\title{
MAGNETIC RESONANCE IMAGING EVALUATION OF SPINAL TUMORS AND ITS COMPARISON WITH HISTOPATHOLOGICAL FINDINGS
}

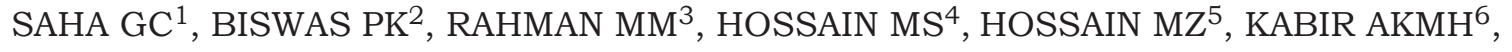 \\ ALAM $F^{7}$, PAUL $T^{8}$
}

\begin{abstract}
:
Objective: The objective of the study was to assess the diagnostic usefulness of MRI in evaluation of spinal tumors.

Methodology: This cross-sectional study was carried out in Dhaka Medical College Hospital, Dhaka. The data was collected from July 2011 to June 2013 and total 51 patients were included in the study. Data was collected from MRI diagnosed spinal tumors who attended at Radiology and Imaging department of DMCH from OPD and indoor patients. Sensitivity, specificity, positive predictive value, negative predictive value, and accuracy of MRI for evaluation of spinal tumors were detected.

Results: Out of 51 patients 26 (51\%) was intradural extramedullary, 13 (25.5\%) was extradural and 12 (23.5\%) was intramedullary. Distribution of patients according to MR diagnosis. Among the 51 patients 40 were diagnosed spinal tumor and 11 were not spinal tumor by MRI. Among the 40 spinal tumuor diagnosed 12 (23.5\%) were schwannoma, 02 (3.9\%) were neuro fibroma, $11(21.6 \%)$ were meningioma, 07(13.7\%) were ependymoma, 05(9.8\%) were astrocytoma, 02(3.9\%) were metastasis and 1 (2.0\%) was osteoblastoma. Out of all cases 40 were diagnosed as spinal tumour by MRI and among them 39 were confirmed by histopathological evaluation. They were true positive. One case was diagnosed as having spinal tumour by MRI but not confirmed by histopathological findings. That was false positive. Out of 11 cases of non tumour which were confirmed by MRI, 3 were confirmed as spinal tumour and 8 were non-tumour by histopathological findings. They were false negative and true negative respectively. Sensitivity, specificity, positive predictive value, negative predictive value and accuracy of the MRI in the diagnosis of spinal tumour were $92.86 \%, 88.89 \%, 97.50 \%, 72.73 \%$ and $92.15 \%$ respectively.

Conclusion: The present study conducted to assess the diagnostic usefulness of MRI in evaluation of spinal tumors among the Bangladeshi population. Study revealed high sensitivity, specificity and accuracy of the MRI in the diagnosis of spinal tumour. MRI should be the initial procedure in the evaluation of suspected tumors of the spine.
\end{abstract}

J Dhaka Med Coll. 2017; 26(2) : 162-166

\section{Introduction:}

Spinal tumors are not uncommon in our country. Spinal tumors can cause significant morbidity. Primary spinal cord tumors constitute $0.5-0.65 \%$ of all tumors. Spinal tumors can be classified as intramedullary, intradural extramedullary and extradural. (Chamberlain and tredway 2011) ${ }^{1}$.

MRI plays a central role in the visualization of spinal tumors, easily allowing tumors to be classified as extradural, intradural extramedullary, or intramedullary which is very

1. Dr. Gopal Chandra Saha' Assistant Professor, Department of Radiology and Imaging, Institute of Child and Mother Health, Dhaka.

2. Dr. Prodip Kumar Biswas, Associate Professor of Medicine, Dhaka Medical College, Dhaka

3. Dr. Md. Motlabur Rahman, Associate Professor of Medicine, Dhaka Medical College, Dhaka

4. Dr. Mohammed Shahadat Hossain, Assistant Professor of Medicine, OSD, DGHS. Attached to Faridpur Medical College, Faridpur.

5. Dr. Mohammad Zaid Hossain, Associate Professor of Medicine, Dhaka Medical College, Dhaka.

6. Dr. A.K.M Humayon Kabir, Associate Professor of Medicine, Dhaka Medical College, Dhaka

7. Dr. Ferdous Alam, Assistant Professor of Surgery, BSMMU

8. Dr. Tushit Paul, Honorary Medical Officer, Dept. of Medicine, Dhaka Medical College, Hospital, Dhaka

Correspondence : Dr. Gopal Chandra Saha Assistant Professor, Department of Radiology and Imaging, Institute of Child and Mother Health, Dhaka.

Received: 10 May 2017

Revision: 06 August 2017

Accepted: 25 August 2017 
useful in tumor characterization. Intradural tumors; the majority is extramedullary, with nerve sheath tumors being the most frequent. (Struffert et al. 2004) ${ }^{2}$.

The role of MRI for the evaluation of the spine is expanding rapidly. In addition to being noninvasive, MRI offers high soft-tissue contrast and multiplanar imaging capability. MRI examinations of the spine usually include a T1weighted spin-echo and a T2-weighted spinecho and/or a gradient-echo sequence (Berns et al. 1989)3.

Recently, development has been made in different types of health services in Bangladesh. Several health care institutions for the management of spinal tumours have already been established in Dhaka and other part of the country both in private and public sectors. Though the exact statistics of incidence and prevalence of spinal tumour in our country is not available but it is the common opinion that the number of such kind of problem is increasing day by day. Appropriate diagnostic procedure should always need upgrade for better management of the spinal tumour. Dhaka Medical College (DMC) is a tertiary level Hospital in Bangladesh. Sophisticated technology and multidimensional services have been added a new dimension in this hospital for health care services. Department of Radiology and Imaging is one of the richest departments in this Hospital. So, to do this kind of study, I think $\mathrm{DMC}$ is an ideal place.

\section{Materials and methods:}

This cross-sectional study was carried out on 54 patients from 11-70 yrs of age, referred for MRI of spine with a clinical suspicion of intraspinal tumour to radiology and imaging department of Dhaka medical college Hospital, Dhaka from July 2011 to June 2013 after approval of ethical committee. Three cases were excluded from the study due to unavailability of histopathological report in two patients and other one refused to do operation. Finally, 51 patients were included in the study. Patients with clinically suspected spinal tumors referred to Radiology \& Imaging department of $\mathrm{DMCH}$ either by OPD or Inpatient department of Neurosurgery, Neuromedicine and orthopedics were selected as cases. Estimated 51 patients fulfil the inclusion criteria and were evaluated. Sampling technique was purposive sampling. Appropriate data was collected by using a preformed data sheet. . All other needed data was collected from history sheet and investigation papers. After tissue biopsy all histopathological reports were collected. Statistical analysis of the results was done by using computer based statistical software, SPSS (SPSS Inc, Chicago, IL, USA).

\section{Results:}

Among the 51 patients 32 (62.7\%) were male and $19(37.3 \%)$ were female. Male to female ratio is $1.68: 1$.

Among 51 patients $04(07.8 \%)$ were in the age group of d"20 years, $07(13.7 \%)$ were in the age group of 21 to 30 years, $14(27.5 \%)$ were in the age group of 31 to 40 years, $18(35.3 \%)$ were in the age group $41-50$ years, $5(9.8 \%)$ were in the age group of 51 to 60 years and rest $3(5.9 \%)$ were in the age group of $>60$ years. The mean age \pm SD is $39.02 \pm 11.63$ years with a range of $13-65$ years.

Among the patients location of tumour of 26 $(51 \%)$ was intradural extramedullary, 13 $(25.5 \%)$ was extradural and $12(23.5 \%)$ was intramedullary.

Among the 51 patients 40 were diagnosed spinal tumour and 11 were not spinal tumour by MRI. Among the 40 spinal tumour diagnosed $12(23.5 \%)$ were schwannoma, $02(3.9 \%)$ were neuro fibroma, $11(21.6 \%)$ were meningioma, 07(13.7\%) were ependymoma, 05(9.8\%) were astrocytoma, 02(3.9\%) were metastasis and 1 $(2.0 \%)$ was osteoblastoma.

Among the 11 patients diagnosed other than spinal tumour by MRI $3(5.88 \%)$ were sequestrated disc, $4(7.84 \%)$ were tubercular spondylitis, 3 (5.88\%) were epidural abscess and rest $1(1.96 \%)$ was subdural Haematoma.

According to histopathological diagnosis among the 51 patients 42 were diagnosed spinal tumour and 9 were not spinal tumour by histopathology. Among the 42 spinal tumour diagnosed $12(23.5 \%)$ were schwannoma, 02 $(3.9 \%)$ were neuro fibroma, $11(21.6 \%)$ were meningioma, 07(13.7\%) were ependymoma, 
05(9.8\%) were astrocytoma, 04(7.8\%) were metastasis and $1(2.0 \%)$ was osteoblastoma.

Among the 9 patients diagnosed other than spinal tumour by histopathology $3(5.88 \%)$ were sequestrated disc, 4(7.84\%) were tubercular spondylitis and rest $2(3.92 \%)$ were epidural abscess.

Out of all cases 40 were diagnosed as spinal tumour by MRI and among them 39 were confirmed by histopathological evaluation. They were true positive. One case was diagnosed as having spinal tumour by MRI but not confirmed by histopathological findings. That was false positive. Out of 11 cases of non tumour which were confirmed by MRI, 3 were confirmed as spinal tumour and 8 were non-tumour by histopathological findings. They were false negative and true negative respectively.

The validity test Sensitivity, specificity, positive predictive value, negative predictive value and accuracy of the MRI in the diagnosis of spinal tumour were $92.86 \%, 88.89 \%, 97.50 \%, 72.73 \%$ and $92.15 \%$ respectively.

Table I

Validity of MRI diagnosis of spinal tumors.

\begin{tabular}{lccc}
\hline MRI & \multicolumn{2}{c}{ Histopathology } & Total \\
& Positive & Negative & \\
\hline Positive & $39(92.9)^{*}$ & 01(11.1) & $40(78.4)$ \\
Negative & $03(07.1)$ & $08(88.9)$ & $11(21.6)$ \\
Total & $42(100.0)$ & $09(100.0)$ & $51(100.0)$ \\
\hline
\end{tabular}

*Figure within parentheses indicates column percentage.

Table II

Validity test

\begin{tabular}{lc}
\hline Validity test & Value (\%) \\
\hline Sensitivity & 92.86 \\
Specificity & 88.89 \\
PPV & 97.50 \\
NPV & 72.73 \\
Accuracy & 92.15 \\
\hline
\end{tabular}

\section{Discussion:}

Primary spinal cord tumors constitute $2 \%$ to $4 \%$ of all central nervous system neoplasms and are characterized based on their location as intramedullary, intradural extramedullary, and extradural. Metastases, lymphoma, and multiple myeloma are the most frequent malignant spinal tumors. Primary osseous tumors of the spine, in contrast, are rare conditions but may demonstrate typical imaging findings. A correct and complete diagnosis of neurologic symptoms is necessary to give appropriate treatment (Bilsky et al. 1999) ${ }^{4}$.

In the present study among the 51 patients 32 $(62.7 \%)$ were male and $19(37.3 \%)$ were female. Male to female ratio is $1.68: 1$. Avramov et al. $(2009)^{5}$ in a study of 122 patients with reported that $54.0 \%$ were male and $46.0 \%$ were female with male to female ratio was $1.18: 1$

In the present study the mean age $\pm \mathrm{SD}$ was $39.02 \pm 11.63$ years with a range of 13 to 65 years Among them 04(07.8\%) were in the age group of d"20 years, $07(13.7 \%)$ were in the age group of 21 to 30 years, $14(27.5 \%)$ were in the age group of 31 to 40 years, $18(35.3 \%)$ were in the age group $41-50$ years, $5(9.8 \%)$ were in the age group of 51 to 60 years and rest $3(5.9 \%)$ were in the age group of $>60$ years (Table II). The average follow-up period was 23.8 months. The mean patient age was 46.6 years. Campello et al. $(2009)^{6}$ in a recent study of 70 patients with intramedullary spinal cord tumors (IMSCTs) showed that the median age at presentation was 41 years with a range of 18 to 47 years.

Primary spinal cord tumors constitute $2 \%$ to $4 \%$ of all central nervous system neoplasms and are characterized based on their location as intramedullary, intradural extramedullary, and extradural. Characteristic magnetic resonance imaging findings are helpful for localization and characterization of these lesions before treatment, as well as for follow-up after treatment (Beall et al. 2007) ${ }^{7}$. Among the patients location of tumour of $26(51.0 \%)$ was intradural extramedullary, $13(25.5 \%)$ was extradural and $12(23.5 \%)$ was intramedullary. Among 51 patients 15 (29.4\%) had enlarged CSF spaces and $36(70.6 \%)$ had not. Forty-six $(90.2 \%)$ had cord compression and 05 (9.8\%) had not. They reported that MR correctly assigned one of three compartments to all lesions, 9 intramedullary, 14 intradural 
extramedullary (6 schwannomas, 3 neurofibromas, 5 meningiomas), and 6 extradural ( 3 schwannomas, 1 meningioma, 1 cavernous hemangioma, 1 metastatic renal cell carcinoma).

Characteristic magnetic resonance imaging findings are helpful for localization and characterization of these lesions before treatment, as well as for follow-up after treatment (Beall et al. 2007) ${ }^{7}$. According to contrast enhancement $4(07.8 \%)$ had nonenhancing lesion and 47 (92.2\%) had enhancing lesion. Enhancing lesion heterogeneous, homogeneous and ring enhancement were 15(29.4\%), 27(52.9\%) and 5 (9.8\%) respectively.

Diagnosis of a primary spinal cord tumor requires a high index of suspicion based upon clinical signs and symptoms as well as spinedirected MRI (Chamberlain and Tredway 2011) ${ }^{1}$. The category of extramedullary intradural masses includes a variety of lesions from meningiomas and nerve sheath tumors (neurofibromas, schwannomas) to less common tumors (hemangiopericytoma), metastases, benign tumors (lipoma, dermoid, epidermoid), inflammatory disorders (arachnoid adhesions, sarcoidosis), vascular lesions (spinal-dural arteriovenous fistula), and cystic lesions (perineural or Tarlov cysts) (Beall et al. 2007) ${ }^{7}$. Among the 51 patients in the present study 40 were diagnosed spinal tumour and 11 were not spinal tumour by MRI. Among the 40 spinal tumour diagnosed 12(23.5\%) were schwannoma, $02(3.9 \%)$ were neuro fibroma, $11(21.6 \%)$ were meningioma, $07(13.7 \%)$ were ependymoma, 05(9.8\%) were astrocytoma, $02(3.9 \%)$ were metastasis and $1(2.0 \%)$ was osteoblastoma. Among the 11 patients diagnosed other than spinal tumour by MRI 3 $(27.3 \%)$ were sequestrated disc, $4(36.3 \%)$ were tubercular spondylitis, $3(27.3 \%)$ were epidural abscess and rest 1 (9.1\%) was intradural Haematoma (Table XI).

Histopathological diagnosis of 42 was spinal tumour and 9 were not spinal tumour. Among the 42 spinal tumour diagnosed $12(23.5 \%)$ were schwannoma, $02(3.9 \%)$ were neuro fibroma, $11(21.6 \%)$ were meningioma, 07(13.7\%) were ependymoma, 05(9.8\%) were astrocytoma,
$04(7.8 \%)$ were metastasis and $1(2.0 \%)$ was osteoblastoma (Table XII). Among the 9 patients diagnosed other than spinal tumour by histopathology, $3(33.3 \%)$ were sequestrated disc, $4(44.5 \%)$ were tubercular spondylitis and rest $2(22.2 \%)$ were epidural abscess.

Out of all cases 40 were diagnosed as spinal tumour by MRI and among them 39 were confirmed by histopathological evaluation. They were true positive. One case was diagnosed as having spinal tumour by MRI but not confirmed by histopathological findings. That was false positive. Out of 11 cases of non tumour which were confirmed by MRI, 3 were confirmed as spinal tumour and 8 were non-tumour by histopathological findings. They were false negative and true negative respectively.

In the present study sensitivity, specificity, positive predictive value, negative predictive value and accuracy of the MRI in the diagnosis of spinal tumour were $92.86 \%, 88.89 \%, 97.50 \%$, $72.73 \%$ and $92.15 \%$ respectively.

\section{Summary:}

The present cross sectional study was conducted in the Department of Radiology \& Imaging in collaboration with Department of Neurosurgery DMCH \& Department of Pathology, Dhaka Medical College, Dhaka, Bangladesh between the periods of July 2011 to June 2013 to assess the diagnostic usefulness of MRI in evaluation of spinal tumors. Total 51 patients were included in this study. The summary of the study as follows:

- In the present study $32(62.7 \%)$ were male and $19(37.3 \%)$ were female. Male to female ratio is $1.68: 1$. The mean $\pm \mathrm{SD}$ of age was $39.02 \pm 11.63$ years with a range of $13-65$ years. Four $(07.8 \%)$ were in the age group of d"20 years, $07(13.7 \%)$ were in the age group of 21 to 30 years, $14(27.5 \%)$ were in the age group of 31 to 40 years, $18(35.3 \%)$ were in the age group 41-50 years, 5 (9.8\%) were in the age group of 51 to 60 years and rest $3(5.9 \%)$ were in the age group of $>60$ years.

- Most common presenting symptoms was back pain $(98.0 \%)$. Limb weakness was the presenting symptoms in $45(88.2 \%)$ 
patients. Other presenting symptoms loss of bowel and bladder control, loss of sensation and paraplegia were $14(27.5 \%)$, $14(27.5 \%)$ and $12(23.5 \%)$ respectively. Among the patients location of tumour of $26(51.0 \%)$ was intradural extramedullary, $13(25.5 \%)$ was extradural and $12(23.5 \%)$ was intramedullary.

- $\quad$ Fifteen (29.4\%) had enlarged CSF spaces and $36(70.6 \%)$ had not. Among 51 patients $46(90.2 \%)$ had cord compression and 05 (9.8\%) had not. Among 51 patients 12 $(23.5 \%)$ had swelling of spinal cord compression and $39(76.5 \%)$ had not. Among the 51 patients $4(07.8 \%)$ had non enhancing lesion and $47(92.2 \%)$ had enhancing lesion. Enhancing lesion heterogeneous, homogeneous and ring enhancement were 15(29.4\%), 27(52.9\%) and $5(9.8 \%)$ respectively.

- Out of 51 patients, 40 were diagnosed spinal tumour and 11 were not spinal tumour by MRI. Among the 40 spinal tumour diagnosed $12(23.5 \%)$ were schwannoma, 02 (3.9\%) were neuro fibroma, $11(21.6 \%)$ were meningioma, 07(13.7\%) were ependymoma, 05(9.8\%) were astrocytoma, 02(3.9\%) were metastasis and $1(2.0 \%)$ was osteoblastoma. Among the 11 patients diagnosed other than spinal tumour by MRI, 3 (27.3\%) were sequestrated disc, 4(36.3\%) were tubercular spondylitis, $3(27.3 \%)$ were epidural abscess and rest $1(9.1 \%)$ was intradural Haematoma.

- Among the 51 patients 42 were diagnosed spinal tumour and 9 were not spinal tumour by histopathology. Among the 42 spinal tumour diagnosed by histopathology, $12(23.5 \%)$ were schwannoma, 02 (3.9\%) were neuro fibroma, $11(21.6 \%)$ were meningioma, 07(13.7\%) were ependymoma, 05(9.8\%) were astrocytoma, 04(7.8\%) were metastasis and $1(2.0 \%)$ was osteoblastoma. Among the 9 patients diagnosed other than spinal tumour by histopathology, 3 (33.3\%) were sequestrated disc, $4(44.5 \%)$ were tubercular spondylitis and rest $2(22.2 \%)$ were epidural abscess.
Out of 51 patients, 40 were diagnosed as spinal tumour by MRI and among them 39 were confirmed by histopathological evaluation. They were true positive. One case was diagnosed as having spinal tumour by MRI but not confirmed by histopathological findings. That was false positive. Out of 11 cases of non tumour which were confirmed by MRI, 3 were confirmed as spinal tumour and 8 were non-tumour by histopathological findings. They were false negative and true negative respectively. Sensitivity, specificity, positive predictive value, negative predictive value and accuracy of the MRI in the diagnosis of spinal tumour were $92.86 \%, 88.89 \%, 97.50 \%, 72.73 \%$ and $92.15 \%$ respectively.

\section{Conclusion:}

The present study conducted to assess the diagnostic usefulness of MRI in evaluation of spinal tumors among the Bangladeshi population. Study revealed high sensitivity, specificity and accuracy of the MRI in the diagnosis of spinal tumour. MRI should be the initial procedure in the evaluation of suspected tumors of the spine.

\section{References:}

1. Chamberlain MC and Tredway TL (2011). Adult Primary Intradural Spinal cord Tumors: A Review. Curr Neurol Neurosci Rep,11,320-328.

2. Struffert T, Grunwald I, Roth C, Reith W (2004). Spinal intradural tumors. Radiologe, 44(12),1211-27.

3. Berns DH, Blaser SI, Modic MT (1989). Magnetic resonance imaging of the spine. Clin Orthop Relat Res, (244), 78-100.

4. Bilsky MH, Lis E, Raizer J, Lee H, Boland P (1999). The Diagnosis and Treatment of Metastatic Spinal Tumor. The Oncologist,4(6), 459-469.

5. Avramov T, Kyuchukov G, Kiryakov I, Obreshkov N, Handjiev D, Nedelko R (2009). Results of spinal tumors surgery. Journal of IMAB-Annual Proceeding (Scientific Papers); Book 1:84-88.

6. Campello C, Le Floch A, Parker F (2009). Neuroepithelial intramedullary spinal cord tumors in adults: Study of 70 cases, in American Academy of Neurology Annual Meeting.: Seattle, WA.

7. Beall DP, Googe DJ, Emery RL, Thompson DB, Campbell SE, Ly JQ, DeLone D, Smirniotopoulos J, Lisanti C, Currie TJ (2007). Extramedullary intradural spinal tumors: a pictorial review. Current Probems inl Diagnostic Radiology, 36(5),185-98. 